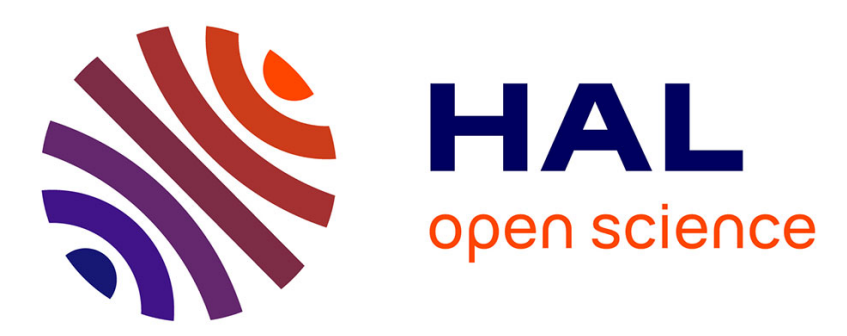

\title{
Le " doyen contrôleur des coûts" : outils de gestion et allocation des ressources dans la gouvernance des universités
}

Pascal Fabre

\section{- To cite this version:}

Pascal Fabre. Le "doyen contrôleur des coûts ": outils de gestion et allocation des ressources dans la gouvernance des universités. La place de la dimension européenne dans la Comptabilité Contrôle Audit, May 2009, Strasbourg, France. pp.CD ROM. halshs-00458929

\section{HAL Id: halshs-00458929 \\ https://shs.hal.science/halshs-00458929}

Submitted on 22 Feb 2010

HAL is a multi-disciplinary open access archive for the deposit and dissemination of scientific research documents, whether they are published or not. The documents may come from teaching and research institutions in France or abroad, or from public or private research centers.
L'archive ouverte pluridisciplinaire HAL, est destinée au dépôt et à la diffusion de documents scientifiques de niveau recherche, publiés ou non, émanant des établissements d'enseignement et de recherche français ou étrangers, des laboratoires publics ou privés. 


\title{
Le « doyen contrôleur des coûts » : outils de gestion et allocation des ressources dans la gouvernance des universités
}

\author{
Pascal FABRE \\ Maître de conférence à l'Université d'Orléans \\ Laboratoire des Collectivités Territoriales (LCT) \\ Domaine universitaire, Rue de Blois, BP 6739 F, 45067 Orléans Cedex 2 \\ pascal.fabre@univ-orleans.fr
}

\begin{abstract}
Résumé
L'implémentation des décisions présidentielles, en particulier celles relatives à la contrainte financière, constitue un défi à la gouvernance des universités. Par rapport à cet objectif, les directeurs d'UFR ou doyens représentent une courroie de transmission vitale entre la présidence et les composantes. L'objectif de cet article est donc d'examiner quels obstacles rencontrent les directeurs d'UFR chargés de décliner la contrainte financière dans leurs établissements et parallèlement d'examiner en quoi les outils de gestion (en particulier la comptabilité analytique) peuvent contribuer à lever (au moins partiellement) ces obstacles.
\end{abstract}

Mots Clés. - Gouvernance - Comptabilité de gestion - Régulation - Planification - Université

Abstract

The implementation of presidential directives, in particular concerning the financial constraint, constitute a challenge for the universities governance. With regard to this aspect, the directors of UFR or deans are a vital drive belt between the presidency and the faculties. The aim of this paper is firstly to study which obstacles meet directors of UFR in charge of financial constraint in their faculties and secondly to understand how the management tools (particularly cost accouting) can contribute to raise these obstacles

Keywords. - Governance - Cost accouting - Régulation - Planification - University

\section{Introduction}

Souvent décrites comme des «anarchies organisées» (Cohen, March et Olsen, 1972), les universités et leurs composantes posent des problèmes spécifiques de gouvernance. Du point de vue de la régulation et du contrôle, contrôler la pertinence des dépenses pour mieux allouer les ressources est un des aspects les plus délicats de cette gouvernance des universités (Baussart, 2005). Il s'agit d'un thème actuel, porteur de forts enjeux, et paradoxalement très peu traité en Sciences de gestion. De plus, si les travaux existants en Sociologie comme en Sciences de Gestion, ont mis l'accent sur la Présidence et le contrôle central, peu de choses ont été écrites sur la façon dont les orientations (en particulier budgétaires) définies par la présidence sont effectivement implémentées aux niveaux secondaires de l'institution. Un tel problème peut a priori être considéré comme secondaire. Toutefois, que le terme gouvernance désigne le gouvernement des universités ou que l'on considère que la gouvernance a pour objet de gouverner les décisions des dirigeants et de délimiter leur latitude décisionnelle (Charreaux, 2002), il faut souligner avec Méla (2007, p. 6) « qu'il ne sert à rien d'avoir le 
pouvoir de décision si on n'a pas celui d'imposer les décisions. C'est pourquoi la question de (savoir) comment implémenter les décisions est le principal défi pour améliorer la gouvernance d'une université ». Or même dans le nouveau cadre défini par la loi de 2007 et la LOLF, les présidents auront besoin de relais pour leurs actions. Même s'ils ne sont pas des alliés naturels de la Présidence (Mignot-Gérard, 2006), les doyens ou directeurs d'UFR n'en constituent pas moins une courroie de transmission vitale entre cette Présidence et les composantes (Musselin, 2001) et il devrait en être à l'identique à l'avenir, même si d'autres structures plus transversales complètent le schéma organisationnel universitaire. Présentement, comme le souligne Trannoy (2006, p. 9) de façon quelque peu brutale "Pour la Présidence, une «bonne UFR » est une UFR qui contient ses coûts dans ses limites imparties et le directeur d'UFR est essentiellement un contrôleur des coûts ». Comment les doyens s'acquittent de cette tache ? Pas très bien, selon la Conférence des Présidents d'Université (2002) «...actuellement, dans nombre d'universités, on peine à imposer cette priorité de l'institution sur les intérêts particuliers. La difficulté rencontrée par nombre d'établissements, pour réguler le volume d'heures complémentaires d'enseignement, par exemple, témoigne de ce tâtonnement entre intérêt général et intérêt de certains groupes ou de certaines personnes ». Parallèlement de nombreuses instances politiques (Sénat, 2007) ou universitaires déplorent l'absence d'outils structurés pouvant être utilisés dans le cadre universitaire pour le contrôle des coûts et la répartition des ressources. Il s'agit donc d'étudier quels obstacles rencontrent les directeurs d'UFR chargés de décliner la contrainte financière dans leurs établissements et parallèlement d'examiner en quoi les outils de gestion (en particulier la comptabilité analytique) peuvent contribuer à lever (au moins partiellement) ces obstacles. L'étude sera centrée sur le contrôle des heures complémentaires qui représente un enjeu fort aussi bien au niveau financier qu'opérationnel. Nous nous appuierons sur trois études de cas choisies pour leur caractère « exemplaire »

1. La fermeture d'un master professionnalisé dans une UFR A ;

2. La création puis l'abandon d'un système de contrôle des coûts dans une UFR B ;

3. L'instauration d'un système de contrôle des coûts dans une UFR C suite à une alternance politique.

Les données ont été recueillies par entretien et recherche documentaire pour les trois UFR (situés dans trois universités différentes) mais également par observation participante pour un des UFR. Ont été interrogés les responsables d'UFR, les vice présidents chargés des moyens ainsi que les enseignants chercheurs ayant participés au processus de mise en place des systèmes de contrôle des coûts mais également, dans la mesure du possible, les responsables des services administratifs (voir annexe 1). Si les situations décrites constituent, à notre avis, des exemples intéressants de processus de contrôle de coûts dans un contexte universitaire, elles ne peuvent être considérées toutefois comme représentatives de l'ensemble des UFR, du fait de la forte culture managériale de la plupart des intervenants et des caractéristiques intrinsèques de ces entités qui les rendent plus facilement gouvernables (selon les services des présidences interrogés). Il s'agit donc probablement de situations " au mieux ». De même la position d'observateur participant de l'auteur dans une des UFR peut constituer un biais méthodologique qui a toutefois été partiellement pallié par des entretiens complémentaires avec d'autres parties prenantes et l'examen d'autres situations à même d'éclairer notre problématique mais dans des universités différentes.

Nous examinerons dans une première partie, en quoi cette mission de contrôle des coûts est nécessaire mais porteuse de risque rendant le passage à l'acte des directeurs d'UFR particulièrement délicat. Une deuxième partie permettra de mieux comprendre le rôle que peuvent avoir les outils de gestion dans cette démarche de contrôle des coûts. La dernière partie sera consacrée à la prise de décision et à la possibilité d'intégrer paramètres techniques issus des outils de gestion et paramètres politiques au sein de la gouvernance des universités. 


\section{1 : La mise à l'agenda : une mission nécessaire mais peu motivante}

La mise à l'agenda d'une démarche de contrôle des coûts dans un contexte universitaire n'est que peu traitée par la littérature professionnelle ou académique. «Savoir, comprendre, décider et agir » sont, par exemple, les grandes étapes définies par Baussart $(2005$, p. 7) pour mettre en place un système de pilotage de l'établissement. Ceci suppose toutefois qu'il y ait un «vouloir »; c'est à dire une volonté politique préalable de s'engager dans un tel type de démarche. Or la situation délicate dans laquelle se trouvent le plus souvent les responsables d'UFR rend le passage à l'acte particulièrement peu évident.

\subsection{Une situation souvent ingérable}

S'il convient d'analyser précisément les causes de la dérive assez fréquente des heures complémentaires (causes internes mais aussi externes aux UFR), il importe également de bien comprendre en quoi le positionnement du directeur d'UFR complique singulièrement son action.

\subsection{1 : Une tendance « naturelle » à la dérive aggravée par l'absence de contrôle de la tutelle}

L'examen du système universitaire permet de constater assez fréquemment des dérives importantes au niveau des heures complémentaires (CPU, 2002). Les consommations d'heures au niveau des composantes sont souvent supérieures aux dotations de celles-ci (ce qui comprend les heures liées aux postes auxquelles s'ajoute le volant d'heures complémentaires alloué à chaque composante par l'université). La première cause tient à des dérapages fréquents relatifs au coût des formations. L'attribution de ressources aux composantes est encore, assez souvent, faite sur des bases San Remo (soit une dotation par étudiant multipliée par le nombre d'étudiants). Bien que le système San Remo ait été abandonné pour l'allocation des moyens aux universités au profit d'une dotation globale, il est souvent conservé pour la répartition interne entre composantes et ensuite entre filières, sans doute faute de mieux mais aussi parce que ce système connu de tous présente une apparence d'équité et de transparence (IGAENR, 2007). Or ces normes San Remo correspondent à des coûts moyens constatés, ce qui ne représente pas forcément l'enveloppe de moyens réellement nécessaire pour fonctionner « convenablement », les heures rémunérant les suivis de stages et les mémoires collectifs n'étant, par exemple, pas pris en compte par ces normes. Ces normes étant considérées comme trop faibles par les enseignants chercheurs, le coût d'une formation a tendance à être supérieur à son «financement» soit le nombre d'étudiants de cette formation que multiplie la dotation horaire par étudiant (le H/E). De plus la contrainte de financement est mal appréhendée par les enseignants chercheurs, d'autant qu'elle ne fait pas l'objet d'un contrôle particulier de l'université ni de la tutelle étatique au moment de la constitution de la maquette. "C'est dans ce contexte que se trouve actuellement l'enseignement supérieur français: on a pris l'habitude de créer de nouvelles filières sans devis estimatif de coût et on incite à modifier celles qui existent sans s'occuper réellement de l'incidence de ces modifications en termes de coût, donc théoriquement, de financement. On ne se réfère plus à des analyses de coûts réels » (Bornarel, 2002, p. 20). Ce manque de contrôle en aval révélé par l'analyse de la littérature a également largement été souligné par les intervenants interrogés, par exemple l'enseignant chercheur chargé du contrôle des coûts 
au sein de l'UFR B : «Au-delà il faut puiser dans la D.G.F. Le moment du contrat, c'est crucial pour recadrer. Après des mécanismes pervers et hyper puissants se mettent en æuvre. Dans l'esprit des enseignants, à partir du moment où on est habilité, à partir du moment où on est dans la maquette, le volume horaire d'un cours devient un droit intangible. Or le ministère se dégage peu à peu de San Remo. Il valide le contenu pédagogique, l'adossement à la recherche, la validité financière relevant de la responsabilité de l'université (selon le principe de l'autonomie des universités). Les présidents d'universités ont laissé passer des revendications infaisables. Après ça revenait au C.A de l'université parce que ce n'était pas tenable financièrement» (Assesseur des coûts, UFR B). Une deuxième cause tient probablement à une maîtrise défectueuse de l'offre de formation. On peut, en effet, constater un processus inflationniste au niveau des créations de diplômes. De nombreux travaux montrent que celles-ci s'opèrent, comme précédemment, sans contrôle efficace de l'université (Biot-Paquerot, 2006) ni de la tutelle étatique. En particulier la procédure de réhabilitation ne s'accompagne pas réellement d'une remise à plat de l'offre et si les créations peuvent s'avérer fort nombreuses, les suppressions de filières s'avèrent rarissimes même en cas de dysfonctionnements avérés (par exemple des effectifs très faibles) (Kletz et Pallez, 2001). L'augmentation de l'offre de formation engendre de façon automatique une augmentation des coûts qui ne s'accompagne pas automatiquement d'une augmentation des recettes correspondantes (liée fréquemment à une augmentation des effectifs). En effet, faute d'une régulation intra-établissement mais également inter-établissement, les phénomènes de cannibalisation ne sont pas rares. L'apport net en étudiants peut s'avérer alors assez faible et les financements additionnels sont loin d'être à la hauteur des coûts supplémentaires engagés (particulièrement dans le contexte actuel de baisse globale des effectifs étudiants). «Document de négociation du projet, le contrat fait une place très insuffisante aux moyens consacrés aux actions programmées par les établissements....On voit ainsi des établissements empiler les années de formation et demander des habilitations en création, sans moyens affichés ou sans redéploiements envisagés de l'offre de formation....Un retour à l'évaluation des coûts des actions envisagées dans les contrats est à brève échéance une démarche inévitable» (Laugenie, 2002, p.23). Des coûts de formation établis sans référence aux possibilités de financement combinés à une offre de formation peu coordonnée et sans doute excessive par rapport au potentiel étudiant aboutissent donc à une consommation excessive d'heures complémentaires eu égard à la dotation allouée par l'université. Comme le souligne Trannoy (2006), la présidence de l'Université se tourne alors vers les directeurs d'UFR et les met en demeure d'économiser les heures complémentaires. Or ceux-ci sont mal armés pour répondre à une telle demande.

\subsection{2 : Un positionnement problématique}

Si le doyen est effectivement le responsable budgétaire de son UFR auprès de la présidence, il est aussi, et peut-être principalement, le défenseur des intérêts de l'UFR auprès de la présidence (Musselin et Friedberg, 1989). Il est élu par ses pairs et non désigné par le président et il est amené à retourner parmi eux au terme de son mandat. Il a d'ailleurs des intérêts particuliers à défendre : son laboratoire, son département, les formations ou il assurait l'essentiel de ses cours et il lui faut penser à gérer au mieux son retour. S'il brigue un autre mandat, il doit, bien entendu, veiller à de pas générer trop d'insatisfaction et de mécontentement. En outre, le pouvoir hiérarchique du doyen reste limité dans son étendue comme dans son application. D'une part, le doyen n'a que peu de prise sur la carrière des enseignants chercheurs et les dispositifs incitatifs qu'il pourrait déployer sont quasi inexistants (Paquet, 2002), d'autre part la culture universitaire privilégie la prise de décision 
consensuelle à l'exercice de l'autorité hiérarchique, ce qui ne favorise pas la prise de décisions difficiles telles la suppression d'une filière de formation ou la réduction de son volume horaire global (Musselin, 2001). Il est donc assez peu enclin à exercer un contrôle par trop directif sur ceux-ci. Pourtant un tel contrôle s'avère souvent indispensable pour faire contrepoint à une vision très autocentrée de beaucoup d'enseignants chercheurs. Si, comme le souligne Musselin (2001), les universités sont en fait des confédérations de micro-unités de production d'enseignement et de recherche, faiblement interdépendantes voire en concurrence les unes avec les autres, un tel raisonnement est aussi valable pour les UFR et engendre une compétition parfois féroce pour l'attribution de ressources, sans prise en compte automatique par les parties prenantes de la situation globale de l'UFR. Cette situation est particulièrement visible dans les UFR $\mathrm{B}$ et $\mathrm{C}$ qui regroupe des départements hétérogènes et très faiblement liés par des activités communes. Les conflits internes pour l'appropriation des ressources (humaines et financières) sont fréquents, certains départements revendiquant d'ailleurs leur autonomie financière. La situation semble beaucoup plus apaisée au sein de l'UFR A, probablement du fait de son caractère monodisciplinaire mais également de l'enchevêtrement de ses activités. En effet beaucoup d'enseignants chercheurs interviennent dans d'autres formations que celles qu'ils dirigent et sont donc intéressés par le maintien et le développement des autres formations.

\section{2 : Un passage à l'acte délicat}

$\mathrm{Vu}$ le positionnement des responsables d'UFR, la meilleure option pour ceux-ci est souvent d'éviter le délicat problème du contrôle des coûts en recherchant des recettes complémentaires. Au vu des cas observés, c'est le plus souvent la conjonction d'une contrainte externe forte et de leurs objectifs propres qui va inciter les directeurs d'UFR à engager finalement ce type de démarche.

\subsection{1 : Des possibilités d'échappatoire réelles mais limitées}

Comme on l'a vu, réduire les coûts s'avère, le plus souvent, pour le responsable d'UFR, un exercice difficile. Extrêmement consommateur de temps, il peut en outre s'avérer dangereux politiquement. Le mieux est donc de ne pas le faire. Une solution évidente est alors de dégager des marges de manœuvres en augmentant les recettes. Faute de latitude sur les droits d'inscription, on ne peut guère jouer que sur la formation continue et l'apprentissage. C'est la stratégie choisie par l'entité A pour laquelle la préoccupation du responsable semble être beaucoup plus de développer les recettes que de contrôler les coûts. "On a $50 \%$ de ressources propres. En effet, je ne peux vivre avec ma dotation. On reçoit des subventions (par exemple du conseil régional pour l'apprentissage) et on a du chiffre d'affaire grâce aux formations à l'étranger et à la collaboration avec les écoles. On ne se limite pas à ce qu'on nous donne. On a un modèle économique... » (Directeur de l'UFR A). De fait, dans l'UFR A, les excédents dégagés par "les activités lucratives » permettent de financer en grande partie les activités de recherche mais aussi le déficit généré par la formation initiale : "...cette dernière activité est obligatoirement déficitaire. Mais on la garde. C'est un choix politique...la formation initiale, c'est la légitimité. C'est politiquement important »(Directeur, UFR A). Dans les entités B et C, le développement de la formation continue est bridée du fait de l'existence de services centraux de formation continue, peu dynamiques commercialement et prélevant, en outre, un fort pourcentage sur toutes les recettes relevant de cette activité. En pratique les recettes de formation continue sont donc marginales et seul le développement de 
l'apprentissage offre des perspectives sérieuses de recettes supplémentaires. Cette dernière possibilité s'avère toutefois limitée, seules certaines formations étant allouables à l'ouverture en apprentissage. Une autre solution consiste à augmenter la dotation budgétaire accordée par l'université soit par la négociation, soit en jouant sur les critères utilisés par l'université pour l'affectation aux composantes. Pour l'entité A, une telle alternative semble exclue, la répartition étant (d'après le directeur de l'UFR A) de nature politique et ne lui laissant que peu de latitude: "J'ai un budget global de un million et demi d'euros (hors prestations internes) or j'ai une dotation de fonctionnement de $25000 €$ à laquelle il faut y ajouter comme ressources les 45 postes d'enseignants chercheurs....La dotation à notre UFR est une enveloppe globale. La répartition est faite de façon politique et n'est pas en notre faveur... On nous dit "Non, non, l'UFR A, vous vous débrouillez! On a $10 \%$ des étudiants mais pas $10 \%$ des moyens. (Directeur d'UFR A). ». Pour les UFR B et C, la dotation aux composantes est effectuée sur la base des dotations San Remo. Celles-ci ne sont pas appliquées directement mais servent à calculer le poids relatif de chaque composante et donc le pourcentage de la dotation globale qui lui sera affecté. Dans ces conditions, la solution évidente est d'augmenter les effectifs mais l'efficacité de cette solution dépend partiellement de paramètres démographiques sur lesquels les universités n'ont aucune prise : "La croissance des effectifs a stoppé et les micro-chapelles, autour des D.E.S.S, se sont cristallisées. On est donc arrivé à des situations du type 12 étudiants pour 700 heures. Toutes les universités ont connu la même situation »(Assesseur des coûts UFR B). Toutefois il est possible, surtout en master 2 d'ouvrir très largement le recrutement, quitte à recruter des candidats n'ayant pas tout à fait le profil requis : «.... il y avait des formations, de troisième cycle notamment, qui n'existaient que par des étudiants étrangers, qui en plus, n'étaient pas très assidus. J'ai au moins dix exemples de DESS ou de DEA, qui ont 8 inscrits, qui avaient 11 candidats; il y en a 2 qui viennent. Et les étudiants en question, sauf un ou deux, on ne les aurait même pas pris en première année de DEUG...» (Biot-Paquerot, 2006, p. 330). On peut également essayer d'augmenter l'attractivité de la composante et donc le nombre d'étudiants par une politique de communication adaptée mais comme le soulignent les responsables de $\mathrm{B}$ et de $\mathrm{C}$ interrogés, les moyens manquent, une grande partie de la DGF étant absorbée par le paiement des heures complémentaires. On peut également essayer d'attirer les étudiants en proposant de nouveaux diplômes mais comme l'ont souligné Kletz et Pallez (2002), une telle solution n'est pas sans risque et peut aboutir à des effets de cannibalisation entre diplômes (l'apport net en étudiants s'avérant relativement faible voire nul). Le phénomène est particulièrement flagrant à l'UFR C, ou il a été amplifié par le fait que les nouvelles spécialités créées présentaient souvent de nombreuses redondances avec d'anciennes spécialités. Une autre solution «classique » et qui peut s'avérer efficace consiste à retarder le processus en puisant sur les réserves. "On peut continuer d'exister même si tout dérape. Envoyer des signaux, c'est se mettre à dos les collègues. Alors pourquoi faire ça? Il y aura toujours de l'argent: les réserves des laboratoires, les réserves de l'université... » (Doyen actuel, UFR C) ; Solution qui n'est pas forcément purement de court terme en fonction du type de gouvernance de l'université....On peut également consacrer aux heures complémentaires l'essentiel de ses ressources propres (taxe d'apprentissage, formation continue, droits d'inscriptions...) quitte à renoncer à tout investissement pédagogique.

\subsection{2 : Une synergie nécessaire entre pressions externes et motivations internes}

Une contradiction existe toutefois. L'observation des UFR A, B et C tend à montrer qu'une démarche de contrôle des coûts peut être engagée alors même que les possibilités d'échappatoires précédemment évoquées existent et sont même mises en œuvre. L'étude de la littérature nous donne quelques pistes à ce sujet. Selon Musselin (1989), c'est souvent une 
situation de crise et de menaces externes (émanant en particulier de l'autorité de tutelle) qui permet l'émergence et l'affirmation d'un style d'action plus directif et qui rend possible des réorientations ou un redéploiement des moyens. Dans les trois UFR observés, une telle pression existe et a probablement joué dans la mise en œuvre effective d'un contrôle des coûts mais sa nature semble assez nettement différente pour chaque composante observée.

Dans l'UFR A, la pression est indirecte et vient du faible poids politique de cette composante au sein de l'université et de la faiblesse des ressources qui lui sont allouées malgré sa forte croissance. Il est alors nécessaire de gérer au mieux les ressources existantes dans une recherche implicite de l'efficience maximale.

Pour l'UFR B, la pression vient à l'origine de la présidence. Celle-ci s'avère d'ailleurs assez interventionniste, étant la seule des trois universités à avoir tenté d'imposer des normes techniques aux composantes (seuil de fermeture à 15 étudiants, volume horaire maximum de 400 heures en face à face....) en complément d'un contrôle budgétaire classique.

Au niveau de l'UFR C, la contrainte exercée par l'université s'avère par contre assez relative. La pression n'est toutefois pas inexistante et vient d'une partie de la base. En effet, le nouveau Doyen a été élu en partie sur la base d'un programme de redressement de la situation financière et la promesse d'une plus grande transparence dans la gestion de la composante au terme d'une campagne très ouverte avec plusieurs candidats (dont l'ancien doyen). Cette pression externe peut, par ailleurs, être instrumentalisée par le directeur d'UFR (Musselin, 1989) qui aura ainsi la possibilité d'invoquer la force majeure et de rejeter la responsabilité de mesures difficiles sur la tutelle. C'est aussi pour lui l'occasion de faire passer des réformes qu'il estime nécessaire sans entamer sa popularité. L'analyse des différentes UFR observées montre en effet qu'il existe des motivations internes puissantes qui vont permettre une appropriation par certains éléments de la composante des objectifs de contrôle et de réduction des coûts : Il s'agit d'abord de recréer des marges de manœuvres financières. Les ressources propres étant largement absorbées par le paiement des heures complémentaires, l'argent manque pour tout le reste : "Beaucoup d'investissements pédagogiques sont «bouffés » (par les heures complémentaires). Ce qui signifie un sous équipement informatique, pas de ressources propres pour le suivi de l'insertion professionnelle des formations longues, pas de cellules de contact avec les entreprises, d'aide à la professionnalisation, pas de politique de communication, pas de développement de l'international ». (Assesseur des coûts de B). De façon plus subtile, il s'agit également de rééquilibrer les activités en faveur de la recherche, en particulier dans les disciplines sous dotées ou le montant individuel d'heures complémentaires assumées par les enseignants chercheurs peut s'avérer très élevé : "Il s'agit de libérer des marges de manœuvres en considérant qu'il y a trop d'heures au point de vue pédagogique et que trop d'heures ça nuit à la recherche. On libère de l'argent pour faire autre chose. On n'abrutit pas les étudiants et on libère du temps pour faire de la recherche ». (Doyen actuel, UFR C). Il s'agit enfin d'optimiser les ressources en allouant celles-ci au plus juste, ce qui permet de créer une réserve de moyens (en particulier humains) pour d'autres activités. C'est en particulier le cas pour A. La combinaison d'une contrainte externe forte et de motivations propres tenant à la gestion interne de leur unité peut donc amener les responsables d'UFR à engager une démarche plus directive de contrôle des couts. Ils vont alors, très souvent, chercher à se doter d'outils comptables et de gestion adaptés.

\section{2 : L'analyse de la situation : des outils à définir}

Comme le souligne Paquet (2002) les universités ne possèdent pas les instruments de pilotage minimum pour assurer correctement leur prise en charge et le contrôle de gestion est inexistant dans la plupart des établissements. Cette situation perdure aujourd'hui (Sénat, 
2007) Or, pour ce qui est des heures complémentaires, le contrôle des coûts est grandement facilité par la mise à disposition d'un outil d'analyse minimum permettant de mesurer la consommation de ressources par formation. Il est ensuite nécessaire de déterminer des normes qui permettent d'une part, d'évaluer la «normalité » de cette consommation de ressources ; d'autre part, de fixer des objectifs de coût mais aussi des objectifs « opérationnels » aux responsables de formations (par exemple en terme d'effectif à atteindre).

\section{1 : S'informer et informer sur les coûts et les marges}

De fait, il n'y a pas de comptabilité analytique obligatoire au niveau des composantes. La décision de mise en place relève de l'initiative individuelle. Généralement, on ne connaît donc pas les coûts par formation, sans parler des coûts par étudiants et par formation. Dans l'U.F.R C, c'est le Responsable des Services Administratifs (RSA) qui a été à l'initiative de la construction du système de comptabilité analytique. Pour l'UFR B, ce fut un enseignant chercheur. Le directeur de l'UFR A n'a pas jugé utile de mettre en place un tel outil du fait de la taille plus réduite de l'UFR et d'une structure de financement différente. Il reste à définir les modalités d'utilisation d'un tel outil.

\subsection{1 : Un outil intéressant d'aide à la négociation}

Contrôler et tenter de réduire les heures complémentaires n'implique pas forcément de disposer d'un outil de comptabilité analytique. Il est possible de se limiter à des mesures générales d'économie (suppression d'abonnements, réduction des heures allouées pour le suivi des stages ou des mémoires collectifs) ou à des mesures forfaitaires touchant indifféremment l'ensemble des formations (réduction de $\mathrm{X} \%$ du volume de chaque cours ou de $\mathrm{Y} \%$ du volume global de chaque formation ou bien exigence d'une économie de $\mathrm{Z}$ heures par formation). Mais, bien entendu, une réduction de $10 \%$ du volume horaire n'aura pas le même effet sur un master doté d'un volume horaire de $300 \mathrm{~h}$ et sur un master avec une dotation de $700 \mathrm{~h}$ et peut avoir des conséquences pédagogiques beaucoup plus néfastes pour le premier que pour le second. On risque donc, faute d'un système d'information adapté, de prendre des mesures trop générales et mal ciblées. De plus ce type de mesures ne prend en compte qu'un aspect du problème: celui du coût des formations. Or celui-ci ne pose réellement problème que si les heures complémentaires de ces formations ne sont pas suffisamment financées, soit par des recettes spécifiques (apprentissage ou formation continue) soit par leur contribution à la dotation globale de fonctionnement (liée pour $\mathrm{B}$ et $\mathrm{C}$ au nombre d'étudiants). En bref, est-il opportun de demander le même effort (à volume horaire égal) à un master comportant une trentaine d'étudiants et générant d'importantes rentrées financières liées à la formation continue et à un master n'incluant qu'une dizaine d'étudiants et sans recettes extérieures ? Le risque est, évidemment, de décourager la recherche d'un meilleur «taux de remplissage» et les initiatives du type «mise en apprentissage » ou développement du public «formation continue ». L'idéal, pour un doyen, est donc de pouvoir disposer d'un compte de résultat par formation mettant en parallèle les coûts spécifiques et les recettes propres de celles-ci. Les problèmes techniques à résoudre sont assez limités. En particulier, il convient de trouver une clé de répartition pour les cours communs. Mais, somme toute, l'outil se révèle facile à mettre en place par les services administratifs de l'UFR. Les comptes de résultat par formation consolidables par département représentent alors pour un doyen un puissant outil d'aide à la négociation. En effet, un coût net par étudiant très élevé est le plus souvent révélateur de dysfonctionnements (une maquette trop lourde se combinant, par exemple, avec un effectif étudiant insuffisant). Les demandes de réduction de coût peuvent alors s'appuyer sur un diagnostic « objectif » et être déterminées 
spécifiquement pour chaque formation en fonction du dépassement. Notons toutefois les limitations des outils mis en place dans les UFR B et C. Il s'agit dans les deux cas de marges sur coût direct avec comme charge principale les frais de personnel enseignant et comme produit les recettes «directement » affectables (dotations San Remo, apprentissage, formation continue...). Or comme l'a très bien analysé Solle (2001), à l'université on reste le plus souvent dans un pilotage postulant l'indépendance des performances des différents services et des différents centres, ce qui est loin d'être avéré. Par exemple, une augmentation de la mutualisation des cours diminue des heures d'enseignement mais complexifie, parfois à outrance, le processus de planification et accroît à terme les besoins en personnel de gestion.

\subsection{2 : Un outil utile mais gênant car révélateur de disparités}

Il reste également à déterminer si les informations sur les coûts ne filtreront que par le biais des face-à-face entre le doyen et les différents responsables pédagogiques de l'U.F.R ou si elles seront largement diffusées à l'intérieur de l'établissement. Le fait de rendre public ou de permettre l'accès à de telles informations présente un inconvénient évident, celui de la visibilité. Ceci est très bien analysé par Bornarel (2002, p.15) «Tout d'abord, le fait d'analyser les coûts en contexte avec tous les acteurs est toujours positif pour ceux-ci mais révélateur des disparités donc gênant. Les évolutions de financement qui devraient s'en déduire, nécessitent souvent un certain courage de la part des gouvernants qui peuvent être soumis aux pressions des groupes de pouvoir. ». En effet, on peut légitimement craindre que la prise de conscience de disparités importantes engendre une augmentation de la conflictualité entre responsables de formations plus ou moins bien dotés et plus ou moins bien gérées mais surtout entre départements composant l'UFR. De plus la publication régulière de tels états va rendre transparente l'action du doyen et mettre éventuellement en évidence son absence d'action ou sa réticence à s'attaquer à certains bastions. Il va alors être possible, dans une certaine mesure, de jauger les actions engagées en fonction de critères objectifs, ce qui rend beaucoup plus difficile la mise en œuvre d'un processus de décision classique basé sur les rapports de force et la négociation (Burlaud, 2004). A cet égard L'UFR C constitue un exemple intéressant. Un calcul sommaire des heures par formation y avait été effectué par le précédent RSA mais il n'a jamais été communiqué au conseil de gestion ni aux enseignants malgré les demandes répétées de certains membres du conseil. L'information était non transparente, ce qui était perçu par ces membres comme une façon pour le doyen précédent de ne pas mettre en évidence les disparités et lui permettre de gérer la situation de gré à gré avec les différents responsables en fonction des rapports de force existants avec un contrôle de type politique (Hofstede, 1981). Il a fallu attendre le changement de Doyen et de RSA pour pouvoir mettre en place une véritable analyse des coûts et surtout que les résultats de cette analyse soient connus de tous. Paradoxalement, une transparence plus grande n'a pas augmenté la conflictualité mais a, semble t'il contribué à la réduire. En effet les enseignants chercheurs étaient globalement très peu informés tant de l'état réel des consommations de ressources que du processus d'allocation de la DGF. Du fait de ce manque d'information, chaque département avait construit sa propre vision des causes des difficultés financières de la composante, celles-ci étant essentiellement de la responsabilité des autres départements. Le diagnostic effectué sur la base de l'outil mis au point par le RSA a clairement montré que les responsabilités étaient partagées et a ainsi contribué à limiter la conflictualité. On retrouve ici les représentations simplifiées bâties par les acteurs dans le cadre d'une situation d'information limitée évoquées par Moisdon (1997). Au sein de l'UFR B, l'information a été largement diffusée mettant en évidence l'importance des disparités. Ceci a contribué à l'évidence à mettre en oeuvre une sorte de pression morale à l'encontre des responsables de formation de B. "... Cela dégageait une marge positive ou négative. Il fallait que ça soit 
équilibré au niveau global (Celui du département). L'outil a été étendu à tout l'UFR en 2003. "Je l'ai mis à disposition de tout le monde pour faire prendre conscience de la sévérité de la contrainte financière ». C'était à eux de prendre leurs responsabilités ». Il ne faut pas oublier que la décision étant fragmentée, le responsable de base ne voit pas les conséquences de ses actions. La comptabilité de gestion rend possible une consolidation progressive des dérapages (spécialité, master, département...) qui permet, dans une certaine mesure, une meilleure prise de conscience des acteurs. "C'était à eux de prendre leurs responsabilités. Ils ont supprimé un certain nombre d'options. On a également beaucoup progressé dans la mutualisation, ce qui n'était pas facile. Cela implique, en effet de coordonner les plannings et de réviser les objectifs pour prendre en compte l'hétérogénéité des publics. La démarche a été incitative et pas impérative. Beaucoup d'entre eux ont travaillé loyalement pour ajuster leur offre. Il y avait une obligation morale. Cela s'est fait. On est passé d'une surconsommation d'heures supplémentaires de 12000 / 15000 à un dépassement de 2000 heures. Une fois habilité, on a continué à travailler.... ».

\subsection{Définir des normes acceptables (de préférence externes)}

S'il n'y a pas de comptabilité analytique obligatoire au niveau des composantes, on peut également remarquer l'absence de standards techniques (volume horaire global par type de diplôme, taille minimale des groupes de TD ou de mémoires) permettant de normer la consommation de ressources. Il en est de même pour ce qui est de la définition d'un effectif minimum pour l'ouverture ou le maintien d'une formation. Il existait précédemment de telles normes définies par la tutelle étatique, en particulier le volume horaire global par type de diplôme. Elles sont également indirectement présentes par l'intermédiaire des normes San Remo, encore très utilisées pour la répartition des ressources aux composantes et qui en tant que coûts standards correspondent à un certain nombre de normes techniques (en particulier l'effectif par formation). En l'absence de directives de l'université, le directeur d'UFR va devoir définir ces normes et (ce qui est sans doute le plus problématique) les faire accepter.

\subsection{1 : La norme imposée : la nécessité d'un pouvoir fort}

Une première possibilité pour le directeur d'UFR est d'imposer des normes techniques de façon à assurer une répartition optimale des ressources. C'est le cas pour ce qui est de l'UFR A, les normes utilisées semblant imposées par la direction. En premier lieu, un montant maximum d'heures est fixé pour les diplômes relevant de la formation initiale : "Un master 2, c'est 300 heures présentielles....C'est fixé. La règle, c'est de rester à volume constant. Mais on peut changer les matières ». En second lieu, l'ouverture d'une formation, même déjà existante, implique la présence d'un effectif minimum. C'est ainsi qu'un master réhabilité n'a pas été ouvert, phénomène très peu fréquent au sein des universités : "On n'a pas ouvert le master parce qu'on n'avait que 5 dossiers. Le minimum c'est 15 étudiants. A moins de 15 étudiants on n'ouvre pas avec une exception pour les formations nouvelles. C'est un coût d'opportunité. Un professeur, c'est plus valable devant 30 étudiants que devant 7 étudiants. » (Directeur, UFR A). Du fait du sous-encadrement structurel de la section, les enseignants permanents sont une ressource rare et intensément utilisée (comme le prouve le nombre très élevé d'heures complémentaires par personne). Cette ressource doit donc être utilisée de façon optimale pour maximiser la «valeur ajoutée sociale » le nombre d'étudiants formés semblant être utilisé comme mesure de l'output. On retrouve ici une notion très proche de la valeur partenariale définie par Charreaux (2000) et utilisée intuitivement par le dirigeant d'UFR même s'il manque ici un «prix d'opportunité » permettant d'évaluer la 
"production». Concernant cette fermeture, aucune résistance n'a été évoquée par le directeur de l'UFR A. A cet égard, il faut encore souligner le caractère beaucoup plus homogène de cet établissement qui regroupe les enseignants chercheurs d'une même section ce qui facilite probablement la convergence des objectifs. D'autre part le nombre d'étudiants est une donnée stratégique et un objectif fédérateur pour l'UFR A qui tente d'augmenter son poids relatif par rapport aux autres composantes.

\subsection{2 : La norme importée : la tentation du rejet}

Dans un cadre universitaire normal, il semble toutefois difficile d'imposer, de façon unilatérale des normes techniques ou des normes de coût. Une solution élégante consiste à les importer, en particulier si celles-ci sont d'origine étatique. Elles vont alors revêtir une apparence d'objectivité, de légitimité qui peut faciliter leur acceptation par les parties prenantes. Cela a été l'option choisie dans l'UFR B ou l'enseignant chercheur interrogé a mis en place un outil qui avait déjà été testé et mis en oeuvre préalablement dans le cadre d'un des départements. On calculait, pour chaque formation, les moyens disponibles calculés sur la base du SAN REMO (H/E x Nombre d'étudiants), ce qui correspondait, de fait, à la dotation réelle de moyens.

Le département en question constituait un "périmètre de solidarité ». Il fallait que l'offre de formation soit équilibrée avec des filières plus richement dotées en étudiants (du fait de leur ancienneté ou de leur attractivité) et génératrices d'excédents, filières finançant les autres filières nouvelles ou moins attractives. L'outil Excel fournissait par formation :

- Les charges : le nombre d'heures de cours

- Les produits : le nombre d'étudiants x le coefficient H/E

Implicitement, le San Remo a été utilisé ici comme norme de consommation de ressource. Il disposait d'un certain nombre d'avantages :

1. il répondait à un besoin de répartition interne

2. il venait de l'extérieur (du ministère) donc il était « légitime »

3. il correspondait à l'outil de répartition interne utilisé par l'université et donc avait un lien fort avec l'apport de ressources par chaque formation

4. il nécessitait un investissement minimum

On retrouve ici un phénomène déjà évoqué par Moisdon (1997). Il y a ici réappropriation d'un outil de gestion par les acteurs pour leurs besoins propres mais hors de son contexte d'origine avec les dangers soulignés par Moisdon. Si la norme est dans un premier temps plus facilement acceptée car dotée d'une légitimité supérieure à une norme imposée, dans un deuxième temps des réactions de rejet sont à craindre lorsque les acteurs appréhendent mieux les décalages éventuels existants entre une norme sortie de son contexte et les consommations réelles. Pour beaucoup, les coûts à l'étudiant du modèle San Remo reposent en effet sur des coûts « constatés » (dans une situation de sous-dotation) et ne constituent pas réellement des coûts « cibles » (Laugenie, 2001).

\subsection{3 : La norme négociée : la difficulté du consensus}

Beaucoup d'auteurs (Mignot-Gerard, 2006) insistent sur la nécessité d'obtenir le consensus des enseignants chercheurs en particulier lorsqu'il s'agit d'outils de gestion portant sur l'allocation des ressources (Abecassis, 2005). C'est la démarche qui a été adopté dans l'UFR C. Le doyen disposait d'un outil similaire à celui de l'UFR B mais de fait, vu le taux de remplissage de beaucoup de formations, le San Remo ne permettait pas de fonctionner correctement et l'alignement des dépenses sur les recettes San Remo n'était pas un objectif 
atteignable à court terme. Il fallait trouver un objectif intermédiaire. Un «comité d'audit» composé de membres du conseil de gestion issus des trois grands départements a donc défini des coûts standards par type de diplôme sur la base de standards techniques. A la base, il s'agissait de définir le volume horaire minimum, une sorte de « ration de survie » permettant de faire tourner une formation «convenablement» (Bornarel, 2002). Le comité d'audit a défini des critères sur une base pédagogique. Toutefois, là aussi, la dotation San Remo a joué le rôle d'une norme de production pédagogique (Trannoy, 2006). En effet le volume horaire de la maquette souhaitable a été calibré de façon à pouvoir arriver à l'équilibre financier avec un nombre « raisonnable » d'étudiants lui-même fixé en fonction de la norme San Remo. Un effectif étudiant à 25 pour un Master 2 a été considéré comme un objectif réalisable. Cet effectif multiplié par un $\mathrm{H} / \mathrm{E}$ de 14 donne un volume horaire de $350 \mathrm{~h}$ acceptable pédagogiquement pour un M2. Ces normes de financement, proposées au doyen, ont ensuite fait l'objet d'un amendement par ses soins de façon à être politiquement acceptables. Les normes fabriquées par le comité d'audit et modifiées par le doyen sont donc très clairement le produit d'un compromis entre une réflexion pédagogique, la contrainte financière issue des normes San Remo et des contraintes politiques. Ceci a, sans doute, facilité leur acceptabilité par les directeurs de départements et les responsables de formation, de même que la composition du comité d'audit qui garantissait dans une certaine mesure «l'objectivité »des travaux. Cette acceptation est toutefois obtenue au prix d'un décalage notable (quoique limité) avec le système de répartition utilisé par l'université C. Les formations respectent (pour la plupart) la norme négociée mais restent assez fréquemment en déficit par rapport à la norme San Remo utilisée par l'Université C, ce que regrette le RSA. Au phénomène de synthèse ascendante évoquée par Moisdon (1997) pour le reporting correspond un phénomène de « répartition descendante » qui contribue à transférer le système de répartition d'un niveau au niveau inférieur pour garder, outre les problèmes de légitimité, une certaine cohérence et une compatibilité avec le système de financement.

\section{3 : La prise de décision : une latitude limitée}

La mise à disposition d'outils de gestion adaptés est un préalable pour le directeur d'UFR mais cela ne lui suffit pas. Il lui faut également pouvoir prendre des décisions difficiles et les faire appliquer. D'autant que les acteurs concernés ne restent généralement pas inactifs et peuvent déployer différentes stratégies pour sauvegarder leur budget d'heures. Or le soutien de la tutelle universitaire ou étatique semble parfois insuffisant pour faire face à celles-ci.

\section{1 : Des actions entravées par des contre mesures très efficaces}

Face aux demandes de réduction des coûts émanant du directeur de l'UFR, les responsables de formation vont déployer un certain nombre de contre-mesures qui peuvent s'avérer particulièrement efficaces. Très bien décrites par Pfeffer et Salancik (1978), elles vont s'exercer sur le mode de la négociation, de l'évitement mais aussi de l'affrontement.

\subsection{1 : Une négociation difficile en amont}

L'étude des cas observés tend à montrer que le contrôle des coûts doit s'effectuer en amont : au moment de la conception des nouvelles maquettes et avant leur habilitation par le ministère. C'est, en effet, un moment privilégié pour faire passer des mesures difficiles. En premier lieu, on peut agir sur le contenu des formations : limitation du volume horaire des 
cours, mutualisation, non ouverture de certaines formations.... Après l'habilitation, il est bien plus difficile de réduire. De plus, la maquette acceptée représente dans l'esprit de beaucoup d'enseignants une sorte de «droit de tirage» or dans les faits, comme on l'a vu précédemment, le ministère donne un avis technique et absolument pas un blanc seing financier. Faute d'outils de gestion, le contrôle par le doyen des maquettes, en face à face avec chaque directeur de formation, est un exercice difficile et très consommateur de temps. Il demande, en outre, une bonne connaissance des formations (par exemple pour apprécier les possibilités de mutualisation) difficile à acquérir d'autant plus que les départements sont diversifiés et éloignés du champ de compétences originel du doyen. "Le plus difficile était de savoir si les enseignements qui faisaient des maquettes correspondaient à un véritable besoin. On venait te dire " et bien ce cours est indispensable! » et tu n'en étais pas sûr. Et puis moi je n'avais pas les moyens de vérifier, évidemment surtout (dans les autres départements). (Dans mon département) je pouvais le faire... » (Ancien Doyen, UFR C). L'asymétrie informationnelle est en faveur des responsables de formation qui pourront en user pour minimiser les efforts à consentir en arguant d'impératifs pédagogiques ou réglementaires. L'existence de coûts standards par type de formation, à l'image de ce qui a été fait à l'UFR C, permet d'éviter cet écueil en fixant un objectif non négociable car déjà négocié. En deuxième lieu, il est également possible d'agir sur l'offre de formation. Supprimer des formations (spécialité ou master) semble, théoriquement, un bon moyen de générer d'importantes économies, plus simple et certainement plus rentable que d'obtenir des économies dans chaque filière. Cependant, comme cela a été souvent souligné, une telle mesure reste exceptionnelle. Il faut, en effet, que cette suppression soit justifiable économiquement, socialement et même pédagogiquement, de par la faiblesse des effectifs concernés. Mais il faut également que cela soit politiquement possible ce qui implique un pouvoir fort (ce qui semble être le cas pour l'UFR A) et/ou une filière à faible enjeu. C'est ainsi que pour A, peu d'enseignants permanents étaient concernés par la fermeture du Master 2, les intervenants étant essentiellement des extérieurs. Pour ce qui est de C, les deux seules formations fermées combinaient un coût élevé et de très faibles effectifs. L'une n'était pas au format LMD, contrairement à d'autres formations avec qui elle faisait doublon. De plus son directeur ne bénéficiait que de peu d'appuis au sein de l'UFR C. L'autre située dans une antenne, ne concernait que marginalement les enseignants chercheurs de C. Notons, à ce sujet, que les pressions politiques (en particulier des élus locaux) empêchent souvent la rationalisation de l'offre universitaire au niveau des antennes.

\subsection{2 : Une épreuve de force inévitable en aval ?}

L'étude des cas observés met également en évidence l'importance du consensus pour éviter les stratégies d'affrontement. Faute de quoi, les acteurs sont à même d'utiliser l'outil politique pour neutraliser un contrôle et une répartition des ressources dont ils contestent le caractère légitime. C'est le cas de l'UFR B pour lequel la situation s'apparente à un véritable retour en arrière. De fait, le système mis en place est abandonné sous la pression de certains responsables de formation pour qui la transparence au niveau de la consommation de ressources s'avère gênante et qui souhaitent revenir à un système de négociation de gré à gré avec le doyen: Lors $d u$ vote $d u$ budget 2007, le Doyen se retrouve confronté à une opposition. La répartition de X, on n'en a rien à faire! San Remo, on n'est pas d'accord! On vote le budget car il met sa démission en jeu et on met en place une commission pour réfléchir à de nouveaux moyens de répartition. On me demande de réfléchir à des critères alternatifs mais l'opposition n'y participe pas et souhaite visiblement revenir à une répartition opaque des moyens. Courant 2007, l'outil n'est pas réactualisé et donc de ce fait abandonné. Pour 2008, le budget se fait sur la base de critères définis par le Doyen et élaboré suite à de 
nombreux face à face ». On touche sans doute là aux limites de la norme importée. Un autre entretien mené avec le nouveau doyen de l'UFR tend, en effet, à montrer que la norme (pourtant largement amendée) était perçue par une partie des enseignants chercheurs comme injuste et favorisant de façon excessive une des composantes de l'UFR. Une norme doit être sans doute être négociée et adaptée au contexte local pour pouvoir être pleinement acceptée ou au minimum les contraintes et situations spécifiques prises en compte de façon à ce qu'elle devienne acceptable par tous (Bernard, 2005). Toutefois il est difficile d'obtenir un consensus total. Dans l'UFR C, la communication n'a sans doute pas été suffisante et l'examen des délibérations du conseil de gestion montre qu'il subsiste de grandes incompréhensions chez certains aussi bien au niveau de la situation de départ que sur l'intérêt de la démarche entreprise (certains ignorant même l'existence d'une démarche de contrôle des coûts). Si les résultats semblent réels en terme de réduction des heures complémentaires non financées, les normes votées par le conseil de gestion ne sont pas toujours respectées par les responsables de filières sous divers prétextes : "On a voté, il y a plus d'un an, des normes qui cadraient ce que devait peser en heures une maquette de M2. On a étudié maquette par maquette et certaines maquettes sont au-delà de ces normes. Il y a des masters avec un léger écart mais certaines formations sont bien au-delà à plus de $20 \%$. On a fait des normes, tout ça pour qu'on puisse déroger sans aucun esprit de solidarité envers les collègues qui les ont respectées » (Doyen actuel, UFR C). Mais les moyens de pression du doyen sont relativement limités et le fait que la tutelle (la présidence) soit perçue par beaucoup d'universitaires comme faible et n'osant pas prendre des décisions brutales, décrédibilise les demandes de celui-ci. Il faut un discours rassembleur (qui existe dans le cas de l'UFR C) mais il manque sans doute la contrainte des procédures extérieures évoquée par Mignot-Gérard (2006) et il est permis de s'interroger sur la nécessité et l'effectivité du support assuré par la tutelle étatique ou universitaire.

\section{2 : Un support insuffisant de la tutelle}

On peut considérer que la tutelle étatique et universitaire met en œuvre deux types de mécanismes de contrôle et de régulation déjà décrits par Lenay et Moisdon (2000) dans leur étude sur les hôpitaux : la régulation et la planification. L'efficacité de ces mécanismes ne semble pas toujours suffisante pour assurer un support effectif au directeur d'UFR dans sa mission de contrôle des coûts. Cette analyse est partagée par les trois vice-présidents chargés des moyens de A, B et C, ceux-ci s'orientant vers un interventionnisme accru de l'université tant au niveau de la définition de normes pédagogiques que de l'élaboration de la carte de formation.

\subsection{1 : une régulation peu efficace faute d'outils et de normes adaptés}

Le mécanisme de contrôle essentiel, dans les cas observés, est une régulation de type économique qui vise à mettre en relation la «production » des établissements et l'attribution de moyens (ancien système San Remo ou futur système Sympa). A l'image de l'Etat (Sénat, 2007), l'Université ne cherche pas à faire une réelle analyse des besoins des composantes ni encore moins à déterminer par formation la consommation de ressources et à jauger la « normalité » de celle-ci. Cette nécessité est transférée aux directeurs de composantes qui ont alors à mettre en place les outils de gestion nécessaires : comptabilité de gestion, coûts standards, normes techniques et financières. Bien qu'ils ne nécessitent pas une forte technicité et que ces outils restent relativement simples, il n'est pas dit que les directeurs d'UFR disposent systématiquement du personnel qualifié nécessaire pour une telle mise en place ou 
qu'ils en perçoivent forcément l'utilité, faute de formation adaptée (Assemblée nationale, 2006). Il n'est pas dit, non plus, que cette situation s'améliore à l'avenir. Certes, de nombreux rapports officiels préconisent la mise en place d'instruments de contrôle de gestion, en particulier la comptabilité analytique. Mais au vu de la littérature administrative actuelle très focalisée sur la mise en œuvre de la LOLF et de la loi LRU on peut craindre un excessif centrage sur des outils comptables de type reporting aux dépens d'outils plus tournés vers l'analyse interne (risque déjà évoqué par Guilhot en 2000 et confirmé par l'enquête de Chatelain-Ponroy en 2006). De plus rien n'est dit sur la déclinaison de ces outils au niveau des composantes et des formations. De toute façon, comme on l'a vu, un tel système a ses limites. La contrainte de coût est ainsi transférée jusqu'au dernier échelon (celui des formations). Mais les responsables de formation, faute de pouvoir transférer la contrainte à leur tour, ont tendance à mettre en œuvre les stratégies protectrices évoquées précédemment. Or, comme on l'a vu, le directeur d'UFR est souvent mal placé pour y faire face, faute d'outils de gestion et de personnels adaptés, par manque aussi des connaissances disciplinaires qu'implique une étude des maquettes, sans compter les paramètres relationnels et l'absence de levier sur les enseignants-chercheurs. "On a atteint les limites de ce que peut fournir le schéma de décentralisation....Le sentiment, c'est qu'aujourd'hui les directeurs nous disent que c'est quant même très compliqué d'être...très rigoureux avec des collègues qu'on croise tous les jours dans les couloirs (Vice président chargé des moyens, UFR C). Une autre modalité de régulation (de type juridique) consiste à fixer au niveau étatique ou au niveau de l'université des normes horaires par type de diplôme. Cette possibilité, déjà mise en œuvre de sa propre initiative par le directeur de l'UFR A est considérée favorablement par certains intervenants interrogés. "Bayrou, c'était en 97. Là, il y avait le cadre qui était fixé (les normes horaires par diplôme). Là il y avait le parapluie...La régulation, c'est pratique...C'est la sérénité puisque tu a un cadre... (Ancien Doyen, UFR C) mais contestée par d'autres "Le CEVU a dit "pas plus de 400 heures par étudiant en face à face. Mais ce n'est pas difficile de trifouiller! (En mettant les heures en cours magistral ou en faisant des groupes réduits en TD). Donc on va introduire de nouvelles règles etc... » (Assesseur des coûts, UFR B). Au delà, la régulation économique qui s'opère au niveau de chaque établissement, peut générer au niveau global des effets pervers non négligeables. C'est le cas, par exemple, pour des formations très ciblées comme certains masters recherche ou d'autre masters à débouchés réels mais restreints. Une prise de décision rationnelle à l'échelon local pourrait amener (en cas de contrainte financière forte) à leur quasi disparition sur l'ensemble du territoire. La nécessité d'une planification assurée par le ministère parallèlement au système actuel et futur de régulation est d'ailleurs clairement évoquée par certaines instances (Sénat, 2007) avec pour objectif d'assurer la cohérence de l'offre globale de formation et de s'assurer du maintien sur le territoire d'un nombre suffisant de formations dans toutes les disciplines.

\subsection{2 : Une planification peu efficace et la nécessité d'une évaluation intra et inter établissement}

Pour reprendre la définition de Lenay et Moisdon (2000, p 27) «La planification...cherche de son coté à définir des pratiques de contrôle fondées sur des autorisations d'activité et d'équipements, des seuils d'activité ou de taille critique, des ratios moyens par spécialité.... ». Il s'agit de répondre à la question suivante : Est-il opportun d'implanter ou de maintenir telle activité par rapport au potentiel humain et aux perspectives d'activité ? La planification, dans le cadre universitaire présente un avantage notable par rapport à la régulation car fondé sur des arguments à caractère pédagogique plus difficilement réfutables par les enseignants chercheurs. En témoignent les échanges assez vifs entre le doyen et les représentants d'un département de l'UFR C au sujet d'un Master Recherche relativement peu coûteux mais avec 
des effectifs nettement inférieurs au seuil de fermeture "préconisé » par le doyen. Ces derniers mettent en avant le caractère prioritaire du Master "C'est stratégique, c'est le cœur.. »Celui-ci leur oppose des arguments de nature pédagogique : la trop grande faiblesse des effectifs ne permet pas des échanges suffisants entre les étudiants et nuit à leur formation. Il faut dire que les choix ne sont pas facilités par l'absence de stratégie formalisée au niveau de l'université. C'est alors au directeur d'UFR que revient la responsabilité de maintenir ou non une formation confidentielle (et donc avec un coût à l'étudiant élevé) mais connectée à un centre de recherche de référence. "Quand on a essayé de faire le ménage pour diminuer les coûts, les formations à faible effectif ont été rapidement pointées du doigt.... Mais qu'est ce qu'on fait sachant que le Centre d'Etude Supérieur $Z$ de renommée internationale engouffre pas mal de moyens et n'a pas beaucoup d'étudiants. C'est une décision de l'UFR ou une décision de la Présidence? »(Assesseur des coûts, UFR B). Actuellement la planification se limite à la procédure d'habilitation ou de réhabilitation des diplômes lors des contrats. Or des coûts excessifs par rapport à la valeur partenariale créé (autrement dit des formations coûteuses et avec un faible effectif) sont souvent le produit de décisions erronées lors de la phase d'habilitation et comme on l'a vu précédemment, le directeur d'UFR est mal placé du fait de sa trop grande proximité avec les apporteurs de projets pour mesurer la pertinence d'une création de diplôme et éventuellement pour s'opposer à sa mise en place. Il ne dispose pas non plus des outils adaptés. Or l'accent mis sur l'évaluation intrinsèque au dépend d'une vision plus large peut conduire à des choix aberrants. On peut citer comme exemple l'habilitation dans l'UFR C d'une spécialité de master après avis favorable du ministère et ceci malgré l'avis très mitigé du doyen et du directeur du département du fait d'un potentiel étudiant très restreint et d'un positionnement peu favorable eu égard à l'offre existante. A cet égard, la tutelle étatique et universitaire ne joue, sans doute, qu'un rôle insuffisant, le dispositif de contractualisation actuel favorisant par trop les critères disciplinaires. Les contraintes de moyens (financiers et humains) ne sont qu'insuffisamment prises en compte, par manque sans doute d'outils analytiques et prévisionnels adaptés. Il en est de même de la capacité des formations à assurer un taux de remplissage suffisant faute d'une évaluation intra et inter établissement permettant la détection des doublons mais aussi la mise en évidence d'une saturation régionale ou nationale des capacités de productions par rapport à la demande potentielle. Surtout l'évaluation ex post fait défaut ou est insuffisamment prise en compte comme en témoigne les difficultés structurelles que l'on rencontre pour fermer une formation. Ceci dit, les VP chargés des moyens interrogés ont conscience des lacunes actuelles de la planification et comptent sur la coopération interuniversitaire pour rationaliser la carte des formations : "La mise en place du PRES avec C permet de travailler sur certains masters en commun...Ce qu'on n'était pas capable de faire tout seul (en terme d'effectifs), on le fait ensemble....L'idée c'est pour partie le PRES et pour certains concours ou certains départements à très petits effectifs, c'est de faire de l'interrégional...Je pense qu'il va falloir dire et bien voila: l'Italien pour A, les Lettres Classiques pour B et l'Allemand pour $C$ » (Vice-président chargé des moyens, Université B).

\section{Conclusion}

Globalement, malgré ces limites, cette recherche permet une réintroduction des outils comptables et de gestion dans la problématique de la gouvernance de l'Université, faisant ainsi contrepoint à l'analyse sociologique. Pour Mignot-Gérard, parce qu'il passe par des alliances avec des « associés-rivaux », parce qu'il agit par 1a persuasion et des formes indirectes de contrôle, le gouvernement de l'université française à la fin de années 1990 semble relever d'un savoir-faire politique qui consiste à gérer des relations de pouvoir et à 
manipuler des arguments, plus qu'il ne repose sur la maittrise de techniques de gestion ou dans l'accumulation de ressources économiques ou sociales. (Mignot-Gérard, 2006). A contrario, l'étude des trois cas précédemment exposés, remet en lumière l'intérêt de disposer d'outils de gestion, même dans des organisations ou le contrôle politique (au sens d'Hofstede) est le mode classique de répartition des ressources. Ceux-ci et en particulier la comptabilité de gestion, présentent l'avantage de permettre un diagnostic partagé par les différentes parties prenantes et semblent donc pouvoir, dans certains cas, réduire la conflictualité, voire même aboutir à une certaine homogénéisation des objectifs. Ils offrent également une certaine protection aux décideurs universitaires contre les pressions politiques internes et facilitent, dans une certaine mesure la prise de décision dans l'affectation des ressources. En conformité avec l'analyse de Charreaux (2000), les outils comptables, seuls, s'avèrent toutefois insuffisants pour optimiser la création de valeur partenariale faute de mesure formalisée satisfaisante de l'output. L'étude permet également résoudre un paradoxe apparent déjà constaté dans d'autres types d'organisation: l'utilisation d'outils de contrôle de type cybernétique au sens d'Hofstede (1981) dans un contexte ou la répartition des ressources ressort plutôt d'un contrôle de type politique comme les mairies ou les universités (Hofstede, 1978). C'est ainsi que les mairies utilisent des grilles de subvention pour répartir automatiquement les subventions sur la base d'une somme d'indicateurs affectés de pondération. Certes quelquefois ces grilles sont imposées mais la plupart du temps elles font l'objet d'une intense négociation préalable avec les parties-prenantes ou sont conçues pour être acceptables par tous. Il en est de même dans le cas de l'utilisation des coûts standards par formation par le directeur de l'UFR C ou là aussi la norme est "négociée ». On est alors très proche de la notion d'environnement négocié développé par Pfeffer et Salancik (1978), l'avantage pour tous les parties prenantes étant d'offrir un cadre normé à la répartition des ressources tout en prenant en compte les spécificités de chacun et en générant d'importantes économies en terme de coûts d'influence. Au bout du compte et pour reprendre les propos de Mignot-Gérard, la maîtrise des techniques de gestion (en particulier la comptabilité de gestion mais aussi les coûts standards) permet sans doute de gérer plus facilement les relations de pouvoir et rend plus aisé la manipulation des arguments car il est sans doute moins difficile de convaincre lorsqu'on peut prouver qu'on a raison. Au-delà de cette observation et en se plaçant au niveau managérial, l'étude, bien qu'elle n'ait aucune prétention à l'exhaustivité, amène à s'interroger sur le peu d'informations relatives à l'implémentation des directives présidentielles comme si cette question allait de soi alors qu'elle peut, de fait, aboutir à une véritable limitation des pouvoirs présidentiels faute de relais opérationnels aux niveaux intermédiaires ; ceci en faisant, on l'a déjà souligné, un véritable enjeu pour la gouvernance de l'université. Or, au vu de la littérature (Chatelain-Ponroy, 2006) et des cas observés, les préoccupations actuelles en terme de recrutement de personnel de soutien et d'outils de gestion semblent être centrées essentiellement sur le reporting LOLF, les problèmes liés à la déclinaison de la stratégie présidentielle et l'amélioration opérationnelle des composantes étant assez peu évoquées. Ceci permet de s'interroger sur la vision même de la gouvernance, sans doute excessivement centrée sur les principaux dirigeants (Charreaux, 2002) au détriment des échelons inférieurs de la structure gouvernementale des entités. 


\section{Annexe 1}

\begin{tabular}{|l|l|}
\hline Objet de l'étude & Personnes interrogés \\
\hline UFR A & Directeur de l'UFR A \\
\hline UFR B & Vice président chargé des moyens (Université A) \\
\hline & Directeur de l'UFR B \\
\hline & Ancien directeur de l'UFR B \\
\hline UFR C & Assesseur des couts de l'UFR B \\
\hline & Directeur de l'UFR C \\
\hline & Ancien directeur de l'UFR C \\
\hline & Vice président chargé des moyens (Université C) \\
\hline & Responsable des services administratifs (UFR C) \\
\hline & Membres du comité d'audit (UFR C) \\
\hline
\end{tabular}

\section{Bibliographie}

Abécassis D. (2005), «Exercer la fonction de directeur d'UFR, la gestion des postes », Ecole supérieure de l'éducation nationale, disponible sur www.esen.education.fr

Assemblée nationale (2006), Rapport d'information $n^{\circ} 3160$ en conclusion des travaux de la mission d'évaluation et de contrôle sur la gouvernance des universités dans le contexte de la LOLF, disponible sur www.assemblée-nationale.fr/12/rap-info/i3160.asp

Baussart H. (2005), "Indicateurs : instruments de pilotage ou outils de contrôle?», Séminaire Amue, «Pilotage de l'établissement : définition et emploi d'indicateurs pertinents ».

Bernard B. (2005), «Les paradoxes des indicateurs émergents », Gérer et Comprendre, N 80, juin, 23-31.

Biot-Paquerot G. (2006), "L'offre de formation des universités : une analyse par la théorie de l'architecture organisationnelle », Doctorat de sciences de gestion, Université de Bourgogne, Dijon.

Bornarel J. (2002), "Financement de l'enseignement supérieur (Formation et Recherche) », Conférence des Doyens et directeur des U.F.R Scientifiques des Universités Françaises (CDUS), Novembre. Disponible sur www.cdus.asso.fr.

Burlaud A, Teller B, Chatelain-Ponroy S, Mignon S, Walliser E. (2004), Contrôle de gestion, Vuibert.

Chatelain-Ponroy S, Rival M, Sponem S, Torset C. (2006), «Les pratiques des établissements d'enseignement supérieur et de recherche en matière de pilotage et de contrôle de gestion; Les outils », Revue Française de Comptabilité, novembre, 43-46.

Charreaux G. (2000), «Gouvernement d'entreprise et comptabilité » in B. Colasse, Encyclopédie de Comptabilité, Contrôle de Gestion et Audit, Economica, 741-756.

Charreaux G. (2002), «Variation sur le thème : à la recherche de nouvelles fondations pour la finance et la gouvernance d'entreprise », Finance, Contrôle, Stratégie, volume 5, N 3, septembre, 5-68. 
Cohen M.D., March J.G. et Olsen J.P. (1972), "A Garbage Can Model of Organizationnal Choice », Admistrative Science Quarterly, 17 (1) : 1-25.

Conférence des présidents d'Université. (2002), Rapport final du groupe de travail de la CPU «Budget global des universités », disponible sur www.cpu.fr

Guilhot B. (2000), «Le contrôle de gestion dans l'université française », Politiques et Management Public, Volume 18, $\mathrm{n}^{\circ} 3$, septembre, 99-119.

Hofstede G. (1981), «Management Control of Public and Not-for-profit Activities », Accounting, Organizations and Society, Vol 6, $\mathrm{n}^{\circ} 3,193-211$.

Hofstede G. (1978), «The Poverty of Management Control Philosophy », Academy of Management Review, juillet, 450-461.

IGAENR (2007), «La mesure de la performance dans le cadre de la mise en auvre de la LOLF dans l'enseignement supérieur », Rapport $\mathrm{n}^{\circ}$ 2007-070, disponible sur www.enseignementsup-recherche.gouv.fr.

Kletz F, Pallez F. (2002), «La constitution de la carte des formations dans les universités : à la recherche d'une stratégie d'établissement », Gérer et Comprendre, $\mathrm{N}^{\circ}$ 67, mars, 16-29.

Laugenie C. (2001), «Evolution du système d'analyse et de répartition des moyens aux établissements d'enseignement supérieur (San Remo) », juillet, disponible sur www.cpu.fr.

Lenay O, Moisdon J.C. (2000), "Croître à l'abri de la gestion ? Le cas du système hospitalier public français », Centre de gestion scientifique, Ecole des mines, Cahier $n^{\circ} 17$, Octobre, disponible sur www-cgs.ensmp.fr.

Méla J F. (2007), "Le principe de subsidiarité dans la gouvernance des universités », Universités et gouvernance, novembre, disponible sur www.jfmela.free.fr .

Mignot-Gerard S. (2006), "Echanger et argumenter. Les dimensions politiques du gouvernement des universités françaises », Doctorat de Sociologie, Institut d'Etudes Politiques, Paris.

Moisdon J C. (1997), Du mode d'existence des outils de gestion, Seli Arslan.

Musselin C, Friedberg E. (1989), «La faiblesse des systèmes de gouvernement des universités », Revue Française de Finances Publiques, $\mathrm{N}^{\circ} 27,185$ - 195.

Musselin C. (2001), «Evolutions organisationnelle des universités », Conférence des Présidents d'Université, Actes du colloque annuel de la conférence : Autonomie des universités, mars, disponible sur www.cpu.fr

Paquet P. (2002), «Faut-il chercher à modéliser le fonctionnement de l'université française ? » in M. Nikitin (éd), Modéliser le fonctionnement des organisations, L'Harmattan, 141-161.

Pfeffer J, Salancik G. (1978), The External Control of Organization, New York, Harper \& Row Publishers.

Sénat $\mathrm{N}^{\circ} 382$ (2007) : groupe de travail sur la réforme de l'allocation des moyens par l'Etat des universités, disponible sur www.senat.fr/rap/r07-382/r07-382.html

Solle G. (2001), «Rénovation des outils de gestion et faits sociaux : le cas des organisations universitaires », Comptabilité Contrôle Audit, Tome 7, mars, 147-159.

Trannoy A. (2006), «Financement des universités, financement des études », Revue d'économie politique, 116 (6) : 745-782. 